\title{
Quantum measurement of photon distribution statistics for intra-cavity EM field monitored by dumping atom-pointer
}

\author{
A. I. Trifanov, E. S. Trifanova \\ ITMO University, St. Petersburg, Russia \\ alextrifanov@gmail.com, etrifanova@gmail.com
}

DOI 10.17586/2220-8054-2017-8-4-447-453

\begin{abstract}
We describe and theoretically study a process of photon distribution statistics measurement for intra-cavity mode of EM field monitored by indirect photo-detection scheme. In particular, we investigate photon number distribution and Mandel's parameter (normalized dispersion) of the mode using statistics of atomic state detector clicks. In our model, a two-level atom-pointer which passes through the cavity interacts with the mode and environment, distorting the measured statistical properties of the mode. To account for this, phase distortion (decoherece) and population relaxation are introduced in the model. In this paper, we use the super-operators approach to intra-cavity mode evolution conditioned by atomic state detector clicks.
\end{abstract}

Keywords: inderect measurements, field statistics, QED interactions.

Received: 25 July 2017

Revised: 02 August 2017

\section{Introduction}

One of the main problems of quantum electromagnetic (EM) field photo-detection is measurement of field states statistical properties using statistics of clicks obtained from detectors and their combinations $[1,2]$. Coherence functions, Q- and P- distributions, phase detection are usually constructed form measured data of homodyne, heterodyne and indirect measurements [3-5]. The most simple existed schemas suggest direct measurement of photon counting rate [6] which allows one to estimate statistics for the photon number operator and reveal coherent properties of the field quantum state such as bunching and anti-bunching.

The conditional photon distribution function for the field depends significantly upon organized the interaction between the EM field and microscopic part of measurement apparatus. Furthermore, relaxation and decoherention due to the existence of environmental modes that may change measurement statistics dramatically [7,8]. Therefore thorough description of such realistic procedure in terms of detectors POVM's is highly desirable [9].

One of the well known ways to control the interaction strength between the field and the photodetector is to use an indirect measurement scheme [10] where a two-level atom-pointer passes through the cavity and interacts with an excited single mode quantum EM field which state is monitored. After that, the state of the atom is detected in the ionizing chamber and the required statistics of intra-cavity mode is restored from atomic state detectors measured data $[7,11]$.

In this paper we use an indirect photodetection scheme to present the protocol for photon distribution statistics measurement [12]. In particular, we suggest monitoring of photon number distribution function and Mandel's parameter from statistics of atomic state detector clicks. Additionally, in the protocol, we account for the non-ideal measurement procedure resulting from the interaction between the atom-pointer and the external environmental modes. The cavity quality factor should be quite high in order to eliminate relaxation processes due to the interaction between intra-cavity and extra-cavity modes.

The work is organized as follows. In the second section we formulate the model of interaction between intra-cavity mode and dumping atom-pointer and obtain a system of differential equations for the elements of atom-field density matrix. After that, in section 3, we introduce a set of conditional evolution superoperators (photodetectors POVM's) and obtain a system of differential equations for them. Section 4 is devoted to solving of this operator-valued system using perturbation theory and the assumption that spontaneous relaxation and excitation rates are small compared to Rabi frequency. In section 5, we use the results of previous section to obtain analytical expressions for mean photon number and Mandel's parameter. Section 6 is for numerical modelling and discussion of obtained results. Section 7 concludes the article. 


\section{The model}

We start with the master equation for the density operator $\rho_{A F}(t)$ of common system of interacting intra-cavity mode and dumped atom:

$$
i \frac{d}{d t} \rho_{A F}(t)=\left[H_{i n t}, \rho_{A F}(t)\right]+i D_{A} \rho_{A F}(t),
$$

where $H_{\text {int }}$ is Hamiltonian describing the interaction between atom and mode. In the interaction picture and under the resonant approximation we can write:

$$
H_{i n t}=\Omega \sigma_{+} a \exp (i \Delta t)+\Omega^{*} \sigma_{-} a^{\dagger} \exp (-i \Delta t) .
$$

Here, $\Omega$ is the Rabi frequency, $\sigma_{+}=|e\rangle\langle g|$ and $\sigma_{-}=|g\rangle\langle e|$ are atomic operators, $a$ is an annihilation operator of cavity mode, $\Delta$ is frequency detuning. The dynamics of the dumping atom may be described in Born-Markov approximation using atomic dissipative part $D_{A}$ in Lindblad form:

$$
2 D_{A} \rho=\gamma_{g e}\left(2 \sigma_{-} \rho \sigma_{+}-\sigma_{+} \sigma_{-} \rho-\rho \sigma_{+} \sigma_{-}\right)+\gamma_{e g}(\text { c.c. }),
$$

where $\gamma_{g e}, \gamma_{e g}$ are the coefficients of spontaneous decay and excitation rates respectively.

System (1) can be written in block-matrix form using the following decomposition for density operator $\rho_{A F}(t)$ :

$$
\rho_{A F}(t)=\sum_{\mu, \nu \in\{g, e\}}|\mu\rangle\langle\nu| \otimes \rho_{\mu \nu}^{F}(t),
$$

where $\rho_{\mu \nu}^{F}(t)$ are taken for matrix elements of the density operator $\rho$ in atomic basis. Then, following [7], we assume that the coherence relaxation time is much shorter than the typical evolution time of the system and set $\Gamma \gg \Omega$. From here, it is easy to show that the following approximation is applicable:

$$
\frac{d}{d t}\left(\rho_{g e}^{F} e^{i \Delta t}\right) \approx \frac{d}{d t}\left(\rho_{e g}^{F} e^{-i \Delta t}\right) \approx 0
$$

Under assumption (5), system (1) may be written as differential equations for blocks $\rho_{\mu \nu}^{F}, \mu, \nu \in\{g, e\}$ where non-diagonal part $\rho_{e g}$ and $\rho_{g e}$ is expressed through diagonal elements $\rho_{g g}^{F}$ and $\rho_{e e}^{F}$. In this way, we obtain closed system of differential equations for diagonal elements (see also [7]):

$$
\begin{aligned}
& \dot{\rho}_{g g}^{F}=\kappa \Gamma\left(2 a^{\dagger} \rho_{e e}^{F} a-\rho_{g g}^{F} a^{\dagger} a-a^{\dagger} a \rho_{g g}^{F}\right)-i \kappa \Delta\left[a^{\dagger} a, \rho_{g g}^{F}\right]+\gamma_{e g} \rho_{e e}^{F}-\gamma_{g e} \rho_{g g}^{F}, \\
& \dot{\rho}_{e e}^{F}=\kappa \Gamma\left(2 a \rho_{e e}^{F} a^{\dagger}-\rho_{g g}^{F} a a^{\dagger}-a a^{\dagger} \rho_{g g}^{F}\right)-i \kappa \Delta\left[a^{\dagger} a, \rho_{e e}^{F}\right]+\gamma_{g e} \rho_{g g}^{F}-\gamma_{e g} \rho_{e e}^{F}
\end{aligned}
$$

where $\kappa=\frac{|\Omega|^{2}}{\Gamma^{2}+\Delta^{2}}$.

\section{Super-operators of conditional evolution}

Just before solving the system (6), we have to give an interpretations for the introduced block-elements $\rho_{\mu \nu}^{F}$. Actually, they correspond to reduced density matrix of the intra-cavity mode conditioned by result of atomic state detection. Particularly, matrix $\rho_{\mu \nu}^{F}$ describes state evolution of the mode when atom prepared in state $|\nu\rangle$ was detected in state $|\mu\rangle$.

Using such interpretation, it is convenient to introduce super-operators of conditional evolution and express them through the set of basis elements of some beautiful algebra. In our case we can notice that maps:

$$
\begin{gathered}
2 K_{0} \rho^{F}=a^{\dagger} a \rho^{F}+\rho^{F} a a^{\dagger}, \quad N \rho^{F}=\left[a^{\dagger} a, \rho^{F}\right], \\
K_{+} \rho^{F}=a^{\dagger} \rho^{F} a, \quad K_{-} \rho^{F}=a \rho^{F} a^{\dagger},
\end{gathered}
$$

obey commutation relations on $S U(1,1)$ algebra with Casimir operator $N$ :

$$
\begin{gathered}
{\left[K_{-}, K_{+}\right]=2 K_{0}, \quad\left[K_{0}, K_{+}\right]=K_{+}, \quad\left[K_{0}, K_{-}\right]=-K_{-},} \\
{\left[K_{0}, N\right]=\left[K_{+}, N\right]=\left[K_{-}, N\right]=0 .}
\end{gathered}
$$

To rewrite system (6) using definitions (7) and (8), we use the following expansion for evolution super-operator of total system:

$$
\left.\rho_{A F}(t)=U(t) \rho_{A F}(0)=\sum_{\mu, \nu \in\{g, e\}} M_{\mu, \nu}(t) \otimes|\mu\rangle\right\rangle\left\langle\langle\nu| \rho_{A F}(0),\right.
$$

where we use notations $|\mu\rangle\rangle\left\langle\left\langle\mu\left|\rho_{A F}=\right| \mu\right\rangle\left\langle\nu\left|\rho_{A F}\right| \nu\right\rangle\langle\mu|\right.$ and $M_{\mu \nu}(t)$ is taken for super-operator of conditional evolution (Kraus operator) of mode state corresponding to the case when atom prepared in state $|\mu\rangle$ was detected in state $|\nu\rangle$. 
Substituting elements $\rho_{g g}^{F}$ and $\rho_{e e}^{F}$ of matrix $\rho_{A F}(t)$ in form (11) into (6) we obtain two independent systems for maps $M_{g g}(t), M_{e g}(t)$ and $M_{g e}(t), M_{e e}(t)$. The first one (which will be the subject of our interest) satisfies the following system:

$$
\left\{\begin{array}{l}
\frac{d}{d t} M_{g g}=-\left(\alpha K_{0}+\beta+\gamma_{g e}\right) M_{g g}+\left(\alpha K_{+}+\gamma_{e g}\right) M_{e g} \\
\frac{d}{d t} M_{e g}=-\left(\alpha K_{0}-\beta+\gamma_{e g}\right) M_{e g}+\left(\alpha K_{-}+\gamma_{g e}\right) M_{g g} .
\end{array}\right.
$$

Here, $\alpha=2 \kappa \Gamma, \beta=-\kappa(i \Delta N+\Gamma)$.

\section{Time evolution of maps $M_{g g}(t)$ and $M_{e g}(t)$}

For simplification of the following discussion, we assume resonance condition $\Delta=0$ for interaction between atom and intra-cavity mode. These immediately give simplified expressions for parameters $\alpha$ and $\beta$ :

$$
\alpha=-2 \beta=2 \frac{\Omega^{2}}{\Gamma} \equiv 2 \Omega \varepsilon,
$$

where $\varepsilon=\Omega / \Gamma \ll 1$. Also, we assume that spontaneous relaxation and excitation rates are small compared to parameters $\alpha$ and $\beta$, which means that

$$
\gamma_{e g}, \gamma_{g e} \ll \varepsilon \Omega,
$$

and apply perturbation theory to solve system (12) up to the first order.

For the zero-order approximation, the solution was found in [5]. Using definitions:

$$
\begin{aligned}
& M_{g}=M_{g g} \exp \left[\left(\alpha K_{0}+\beta+\gamma_{g e}\right) t\right], \\
& M_{e}=M_{e g} \exp \left[\left(\alpha K_{0}-\beta+\gamma_{e g}\right) t\right],
\end{aligned}
$$

and setting $\gamma_{e g}=\gamma_{g e}=0$ system (12) was reduced to exactly solvable one:

with solution of the form:

$$
\left\{\begin{array}{l}
\frac{d}{d t} M_{g}=\alpha K_{+} M_{e} \\
\frac{d}{d t} M_{e}=\alpha K_{-} M_{g}
\end{array}\right.
$$

$$
\begin{gathered}
M_{g}=\cosh \alpha \sqrt{K_{+} K_{-}} t \\
M_{e}=\left(K_{-} K_{+}\right)^{-1} K_{-} \sqrt{K_{+} K_{-}} \sinh \alpha \sqrt{K_{+} K_{-}} t .
\end{gathered}
$$

Using (15) and (16), one rewrite system (12) in the form:

$$
\left\{\begin{array}{l}
\frac{d}{d t} M_{g}=e^{\left(\gamma_{g e}-\gamma_{e g}\right) t}\left(\alpha K_{+}+\gamma_{e g} e^{-\alpha t}\right) M_{e} \\
\frac{d}{d t} M_{e}=e^{\left(\gamma_{g e}-\gamma_{e g}\right) t}\left(\alpha K_{-}+\gamma_{g e} e^{\alpha t}\right) M_{g}
\end{array}\right.
$$

and solution of this system will be obtained using perturbation theory by parameters $\gamma_{e g} / \Omega$ and $\gamma_{g e} / \Omega$ :

$$
\left\{\begin{array}{l}
M_{g}=M_{g}^{(0)}+M_{g}^{(1)}, \\
M_{e}=M_{e}^{(0)}+M_{e}^{(1)},
\end{array}\right.
$$

starting from (18), (19) and with initial conditions $M_{g}(0)=E, \frac{d}{d t} M_{g}(0)=0$.

Using previous results for zero-order terms $M_{g}^{(0)}$ and $M_{e}^{(0)}$ we have:

$$
\left\{\begin{array}{l}
\frac{d}{d t} M_{g}^{(1)}= \\
{\left[\gamma_{e g} e^{-\alpha t}+\left(\alpha K_{+}+\gamma_{e g} e^{-\alpha t}\right)\left(\gamma_{g e}-\gamma_{e g}\right) t\right] M_{e}^{(0)}+\left[\alpha K_{+}+\gamma_{e g} e^{-\alpha t}+\left(\alpha K_{+}+\gamma_{e g} e^{-\alpha t}\right)\left(\gamma_{g e}-\gamma_{e g}\right) t\right] M_{e}^{(1)}} \\
\frac{d}{d t} M_{e}^{(1)}= \\
{\left[\gamma_{g e} e^{\alpha t}-\left(\alpha K_{-}+\gamma_{g e} e^{\alpha t}\right)\left(\gamma_{g e}-\gamma_{e g}\right) t\right] M_{g}^{(0)}+\left[\alpha K_{-}+\gamma_{g e} e^{\alpha t}-\left(\alpha K_{-}+\gamma_{g e} e^{\alpha t}\right)\left(\gamma_{g e}-\gamma_{e g}\right) t\right] M_{g}^{(1)}}
\end{array}\right.
$$


For very short time intervals $t \ll \tau=1 / \Omega$ value $\left(\gamma_{g e}-\gamma_{e g}\right) t$ is negligibly small compared to $\gamma_{g e}$ and $\gamma_{e g}$, which allows elimination of terms $\left(\gamma_{g e}-\gamma_{e g}\right) t M_{e, g}^{(1)}, \gamma_{g e, e g}\left(\gamma_{g e}-\gamma_{e g}\right) t M_{e, g}^{(0)}$ and replacement of terms $\gamma_{e g} e^{-\alpha t} M_{e, g}^{(1)}$ and $\gamma_{g e} e^{\alpha t} M_{e, g}^{(1)}$ by $\gamma_{e g} M_{e, g}^{(1)}$ and $\gamma_{g e} M_{e, g}^{(1)}$ correspondingly. From this, we obtain:

$$
\left\{\begin{array}{l}
\frac{d}{d t} M_{g}^{(1)}=\gamma_{e g} e^{-\alpha t} M_{e}^{(0)}+\left(\alpha K_{+}+\gamma_{e g}\right) M_{e}^{(1)}, \\
\frac{d}{d t} M_{e}^{(0)}=\gamma_{g e} e^{\alpha t} M_{g}^{(0)}+\left(\alpha K_{-}+\gamma_{g e}\right) M_{g}^{(1)} .
\end{array}\right.
$$

This inhomogeneous system may be reduced to a second-order linear differential equation for $M_{g}^{(1)}$ :

$$
\begin{aligned}
\frac{d^{2}}{d t^{2}} M_{g}^{(1)}-\left(\alpha K_{+}+\gamma_{e g}\right)\left(\alpha K_{-}+\gamma_{g e}\right) M_{g}^{(1)} & = \\
& {\left[\alpha \gamma_{g e} K_{+} e^{\alpha t}+\alpha \gamma_{e g} K_{-} e^{-\alpha t}+\gamma_{e g} \gamma_{g e}\right] M_{g}^{(0)}-\alpha \gamma_{e g} e^{-\alpha t} M_{e}^{(0)} }
\end{aligned}
$$

with initial conditions $M_{g}^{(1)}(0)=0$ and $\frac{d M_{g}^{(1)}}{d t}(0)=0$.

Using the fact that $t \ll \tau$, we can expand the solution of (24) by the power series of the form:

$$
M_{g}^{(1)}(t)=A_{0}+A_{1} t+A_{2} t^{2}+\ldots
$$

From the initial conditions, it immediately follows that $A_{0}=A_{1}=0$. To find $A_{2}$, we compare coefficients of the zero-order degree by $t$ in the left and right sides of (24). From here, we obtain:

$$
M_{g}^{(1)}(t)=\frac{1}{2}\left[\alpha \gamma_{e g} K_{-}+\alpha \gamma_{g e} K_{+}+\gamma_{g e} \gamma_{e g}\right] t^{2}+\ldots
$$

Finally, for the map $M_{g g}(t)$, we obtain:

$$
M_{g g}(t)=1-\left(\alpha K_{0}+\beta+\gamma_{g e}\right) t+\frac{1}{2}\left[\alpha^{2} K_{+} K_{-}+\left(\alpha K_{0}+\beta\right)^{2}+\alpha \gamma_{e g} K_{-}+\alpha \gamma_{g e} K_{+}+\gamma_{g e} \gamma_{e g}\right] t^{2}+\ldots
$$

\section{Ground state counting rate}

Formula (27) gives expression for conditional evolution of the intra-cavity field state for the case when atompointer prepared in its ground state $|g\rangle$ after interaction with the mode was detected in the same state. To calculate probability $P_{g g}(t)=\left\langle M_{g g}(t)\right\rangle$ of this event let us calculate corresponded quantities for operators $K_{ \pm}$and $K_{0}$ :

$$
\begin{gathered}
\left\langle K_{-}\right\rangle=\operatorname{Tr}\left(K_{-} \rho\right)=\operatorname{Tr}\left(a \rho a^{\dagger}\right)=\operatorname{Tr}\left(a^{\dagger} a \rho\right)=\left\langle a^{\dagger} a\right\rangle, \\
\left\langle K_{+}\right\rangle=\operatorname{Tr}\left(K_{+} \rho\right)=\operatorname{Tr}\left(a^{\dagger} \rho a\right)=\operatorname{Tr}\left(a a^{\dagger} \rho\right)=\left\langle a a^{\dagger}\right\rangle=\left\langle a^{\dagger} a\right\rangle+1, \\
\left\langle K_{0}\right\rangle=\operatorname{Tr}\left(K_{0} \rho\right)=\frac{1}{2} \operatorname{Tr}\left(a^{\dagger} a \rho+a a^{\dagger} \rho\right)=\left\langle a^{\dagger} a\right\rangle+\frac{1}{2} .
\end{gathered}
$$

Then, for probability $P_{g g}(t)$, we will have:

$$
\begin{aligned}
P_{g g}(t)= & \left\langle M_{g g}(t)\right\rangle=\operatorname{Tr}\left(M_{g g}(t) \rho\right)= \\
& 1-\left(\alpha\left\langle a^{\dagger} a\right\rangle+\gamma_{g e}\right) t+\left[\alpha^{2}\left\langle\left(a^{\dagger} a\right)^{2}\right\rangle+\frac{1}{2}\left(\alpha\left(\gamma_{g e}+\gamma_{e g}\right)\left\langle a^{\dagger} a\right\rangle+\alpha \gamma_{g e}+\gamma_{g e} \gamma_{e g}\right)\right] t^{2}+\ldots
\end{aligned}
$$

or in symmetrical form:

$$
P_{g g}(t)=1-\left(\alpha\left\langle a^{\dagger} a\right\rangle+\gamma_{g e}\right) t+\left[\alpha^{2}\left\langle\left(a^{\dagger} a\right)^{2}\right\rangle+\frac{\alpha}{2} \gamma_{e g}\left\langle a^{\dagger} a\right\rangle+\frac{\alpha}{2} \gamma_{g e}\left\langle a a^{\dagger}\right\rangle+\frac{\gamma_{g e} \gamma_{e g}}{2}\right] t^{2}+\ldots
$$

From this expression, the mean value of photon number operator $\left\langle a^{\dagger} a\right\rangle$ and its square $\left\langle\left(a^{\dagger} a\right)^{2}\right\rangle$ may be obtained by differentiating probability function $P_{g g}(t)$ over $t$ for $t=0$ :

$$
\begin{gathered}
\left\langle a^{\dagger} a\right\rangle=-\frac{1}{\alpha}\left(\frac{d P_{g g}(0)}{d t}+\gamma_{g e}\right), \\
\left\langle\left(a^{\dagger} a\right)^{2}\right\rangle=\frac{1}{2 \alpha^{2}}\left[\frac{d^{2}}{d t^{2}} P_{g g}(0)+\frac{d}{d t} P_{g g}(0)\left(\gamma_{e g}+\gamma_{g e}\right)+\gamma_{g e}\left(\gamma_{g e}-\alpha\right)\right] .
\end{gathered}
$$




\section{Numerical results and discussion}

In this section, we compare obtained analytical results with numerical simulation of photo-detection procedure. Fig. 1 contains this comparison for probability to detect atom in its ground state $|g\rangle$ for Fock state with $n=4$ photons, $\Gamma=10$ and $\gamma_{e g}=\gamma_{g e}=0.5$ in frequency units of $\Omega$. Three cases are under consideration: numerical simulation (blue solid line), zero-order (green doted line) and first-order (red dashed line) approximation of the solution. All three lines are quite close nearby the origin and first order approximation shows familiar behaviour with exact solution on considered time interval.

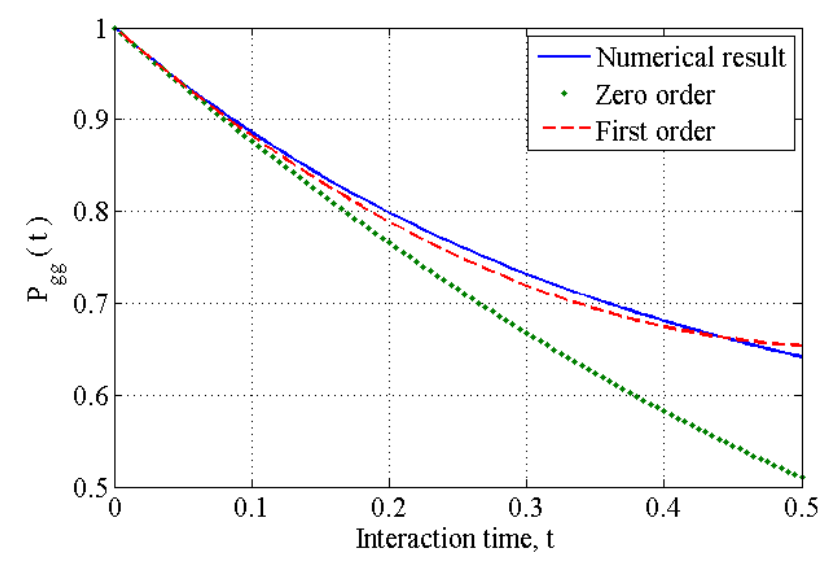

FIG. 1. Probability $P_{g g}$ to detect atom in its ground state: numerical simulation (blue solid line), zero-order (green dotted line) and first-order (red dashed line) approximation

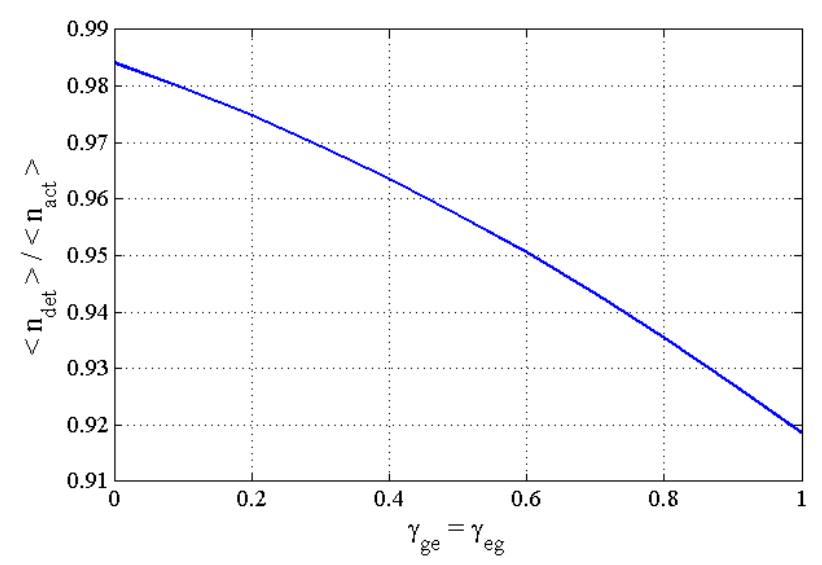

FIG. 2. Relation between actual $n_{\text {act }}$ and detected $n_{\text {det }}$ mean photon number as a function of spontaneous decay and excitation rates

In Fig. 2, we show the relation between actual $n_{\text {act }}$ and detected $n_{\text {det }}$ mean photon number as a function of spontaneous decay and excitation rates for the case $\gamma_{e g}=\gamma_{g e}$ when other parameters are taken the same as in previous figure. All values were calculated for probability function $P_{g g}(t)$ obtained from exact solution. From here, we can see that number of detected intra-cavity photons decrease with increasing spontaneous decay and excitation rates.

Figure 3 shows the relation between actual $n_{\text {act }}$ and detected $n_{\text {det }}$ mean photon number as a function of interaction time between atom and mode prepared in Fock state with $n=4$. Calculations are made for different values of the coefficient of spontaneous excitation $\left(\gamma_{e g}\right.$, first number in the legend) and decay $\left(\gamma_{g e}\right.$, second number in the legend). We can see that curves are grouped in threes according to the same value of $\gamma_{g e}$ which determines accuracy. 


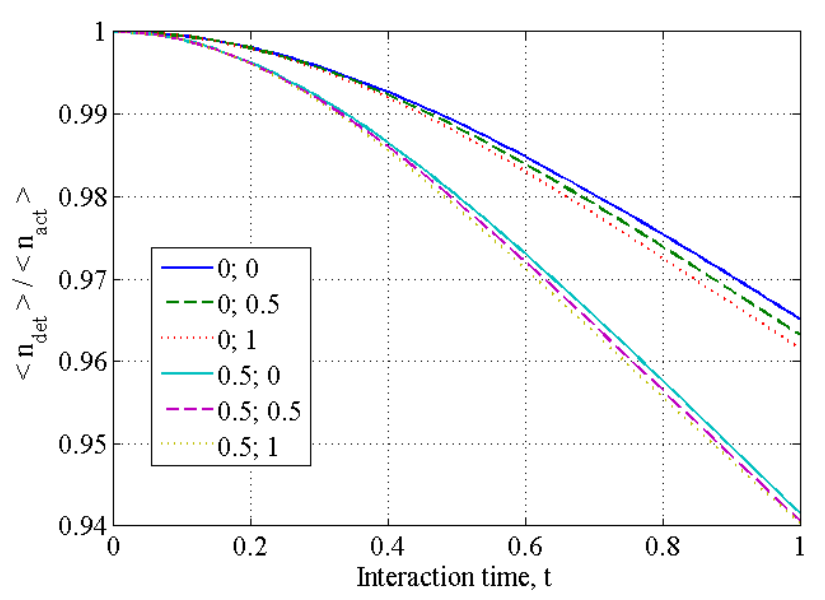

FIG. 3. Relation between actual $n_{\text {act }}$ and detected $n_{\text {det }}$ mean photon number as a function of spontaneous decay and excitation rates

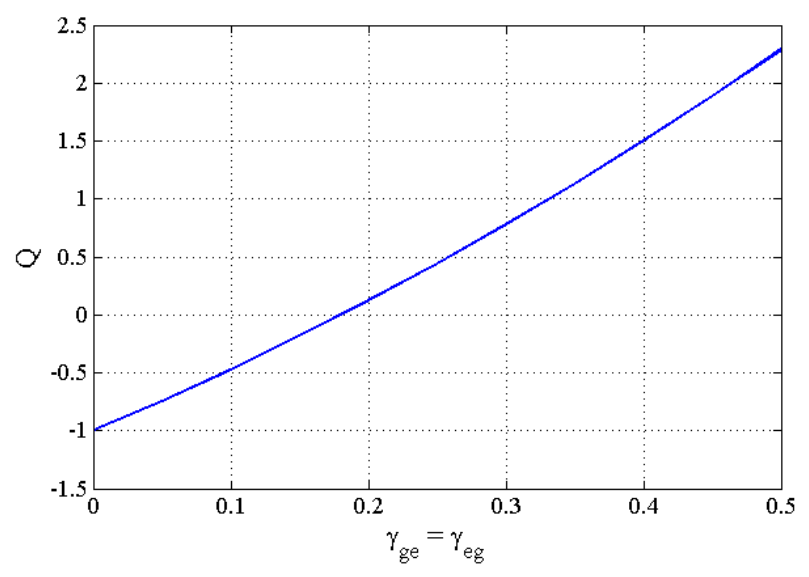

FIG. 4. Estimated value of Mandel's parameter as a function of spontaneous decay and excitation rates

Finally, we investigate estimated value of Mandel's parameter:

$$
Q=\frac{\left\langle n^{2}\right\rangle-\langle n\rangle^{2}}{\langle n\rangle}-1, \quad n=a^{\dagger} a,
$$

as a function of spontaneous decay and excitation rates for the case of initial Fock state with $n=4$ and $\gamma_{e g}=\gamma_{g e}$. When losses are absent, we have typical value $Q=-1$ for the state with definite photon number. For non-zero values of $\gamma_{e g}$ and $\gamma_{g e}$ parameter $Q$ increases reaching the value corresponding to thermal fields. This means that thermal radiation originated from decaying process become predominant.

\section{Conclusion}

In present paper we suggest and analyze an indirect photo-detection protocol of intra-cavity mode statistical properties which may be obtained from statistics of atomic state detector clicks. The obtained probability distribution for detecting atom in its ground state contains, in general, all degrees of photon number operator and allows in principle to restore full statistics of the mode calculating all elements of power series for $P_{g g}$. However, this process is bounded by the finite duration of interaction time between the atom and the mode because calculation accuracy of time derivative is degrades with higher order derivatives.

The estimated number of experiments required for obtaining appropriate statistics for atomic detector clicks at point $t_{0}=0.1 \mathrm{mcs}$ is $N=100$ (where $P_{g g}(0.1)=0.99$ is theoretical estimation). We repeat this cycle of measurements at each point for about 100-200 points and in that way, the overall number of measurement cycles is on the order of $10^{4}$. 
Indirect photo-detection with atom-pointer in use gives beautiful model of quantum measurement due to the possibility of controlling interaction strength and inclusion relaxation process into the model. From this point of view, formulation protocols analogous to well known homodyne and heterodyne detection in terms of indirect model would be very interesting.

\section{Acknowledgements}

This work was partially financially supported by the Government of the Russian Federation (grant 074-U01), by grant MK-5161.2016.1 of the President of the Russian Federation, grant 16-11-10330 of Russian Science Foundation.

\section{References}

[1] Kelley P.L., W.H. Kleiner Theory of electromagnetic field measurement and photo-electron counting. Physical Review, 1964, 136 (2A), P. 316-334.

[2] Braginsky B., Khalili F.Ya. Quantum Measurement, Cambridge University Press, Cambridge, 1992.

[3] Miroshnichenko G.P., Trifanova E.S., Trifanov A.I. An indirect measurement protocol of intracavity mode quadratures dispersion in dynamical Casimir effect. Eur. Phys. J. D, 2015, 69, P. 137.

[4] Smithey D.T., et al, Measurement of the Wigner Distribution and the Density Matrix of a Light Mode Using Optical Homodyne Tomography: Application to Squeezed States and the Vacuum. Phys. Rev Lett., 1993, 70 (8), P. 1244-1247.

[5] Pregnel K.L., Pegg D.T., Binomial states and the phase distribution measurement of weak optical elds. Phys. Rev. A, $2003,67,063814$.

[6] Srinivas M.D., Davies E.B., Photon counting probabilities in quantum optics. Optica Acta, 1981, 28 (7), P. 981-996.

[7] Trifanov A.I., Miroshnichenko G.P. Reduced conditional dynamic of quantum system under indirect quantum measurement. Nanosystems: Physics, Chemistry, Mathematics, 2013, 4 (5), P. 635-647.

[8] Dodonov A.V., Mizrahi S.S. Inclusion of nonidealities in the continuous photodetection model. Phys. Rev. A, $2007,75,013806$.

[9] Mario Ziman, Process positive-operator-valued measure: A mathematical framework for the description of process tomography experiments. Phys. Rev. A, 2008, 77, 062112.

[10] Briegel H.-J., Englert B.-G., Sterpi N., Walther H. One-atom maser: Statistics of detector clicks. Phys. Rev. A, 1994, 49, P. 2962.

[11] Trifanova E.S., Trifanov A.I. Monitoring of quantum mode correlation functions in the presence of pointer state phase relaxation. $J$. of Phys.: Conf. Ser., 2016, 735, 012044.

[12] Johnson D.B., Schieve W.C., Detection statistics in the micromaser. Phys. Rev. A, 2001, 63, 033808. 